The Astrophysical Journal, 569:L61-L64, 2002 April 20

(C) 2002. The American Astronomical Society. All rights reserved. Printed in U.S.A.

\title{
THE PROMPT INVENTORY FROM VERY MASSIVE STARS AND ELEMENTAL ABUNDANCES IN Ly $\alpha$ SYSTEMS
}

\author{
Y.-Z. Qian, ${ }^{1}$ W. L. W. Sargent, ${ }^{2}$ And G. J. Wasserburg ${ }^{3}$ \\ Received 2002 February 19; accepted 2002 March 12; published 2002 March 25
}

\begin{abstract}
It has been proposed that very massive stars (VMSs) dominated heavy-element production until a "metallicity" threshold corresponding to $[\mathrm{Fe} / \mathrm{H}] \approx-3$ was reached. This results in a prompt $(P)$ inventory of elements, the abundances of which were determined from observations of Galactic halo stars with $[\mathrm{Fe} / \mathrm{H}] \approx-3$. We calculate $\Omega_{P}(\mathrm{E})$ from the $P$ inventory for a large number of elements in the intergalactic medium (IGM). Using the available data on $\Omega\left(\mathrm{E}_{\mathrm{ion}}\right)$ for C IV, O VI, and Si IV in Ly $\alpha$ systems, we find that the ionization fractions calculated from $\Omega\left(\mathrm{E}_{\text {ion }}\right) / \Omega_{P}(\mathrm{E})$ are, within reasonable uncertainties, compatible with the values estimated from ionization models for $\operatorname{Ly} \alpha$ systems. This agreement appears to hold from $z \sim 0.09$ to $\sim 4.6$, indicating that the bulk of the baryonic matter remains dispersed with a fixed chemical composition. We conclude that the $P$ inventory was established in the epoch prior to $z \sim 4$.6. The dispersal of processed baryonic matter to the general IGM is considered to be the result of energetic VMS explosions that disrupted baryonic aggregates until the "metallicity" threshold was reached to permit normal astration. The formation of most galaxies is considered to have occurred subsequent to the achievement of this metallicity threshold in the IGM.
\end{abstract}

Subject headings: early universe — intergalactic medium — quasars: absorption lines

\section{INTRODUCTION}

We present predictions for the abundances of a large number of elements in the intergalactic medium (IGM), especially the Ly $\alpha$ systems, based on considerations of a prompt $(P)$ inventory that is dominated by the contributions from very massive $\left(\gtrsim 100 M_{\odot}\right.$ ) stars (VMSs). A comparison will be made between the predictions and the available observational data. The model of the $P$ inventory (Wasserburg \& Qian 2000a; Qian \& Wasserburg 2001a, 2002) claims that, prior to the achievement of the condition corresponding to a metallicity of $[\mathrm{Fe} / \mathrm{H}] \approx-3$ in the IGM, the predominant mechanism of astration is by formation of VMSs. This condition is identified based on the sharp rise in the abundances of the heavy $r$-process elements such as $\mathrm{Ba}$ and $\mathrm{Eu}$ in Galactic halo stars with $[\mathrm{Fe} / \mathrm{H}] \approx-3$ (e.g., McWilliam et al. 1995; Burris et al. 2000). This sharp rise is considered to represent the occurrence of Type II supernovae (SNe II) that result from evolution of normal stars with masses of $\sim 10-60 M_{\odot}$ in the absence of VMSs. Further support for a threshold at $[\mathrm{Fe} / \mathrm{H}] \approx-3$ for normal astration comes from the observation that all damped $\operatorname{Ly} \alpha$ systems with redshift $z \approx 1.5-4.5$ have a minimum $[\mathrm{Fe} / \mathrm{H}]$ of $\approx-2.7$ (e.g., Prochaska \& Wolfe 2000; Prochaska, Gawiser, \& Wolfe 2001). The metallicity of $[\mathrm{Fe} / \mathrm{H}] \approx-3$ is considered to represent the critical condition for sufficient cooling and fragmentation of gas clouds to permit major formation of normal stars with masses of 1-60 $M_{\odot}$ (Wasserburg \& Qian 2000a). This is supported by ab initio calculations of Bromm et al. (2001a), which showed that a critical metallicity of $\sim 5 \times 10^{-4}$ the solar value (corresponding to $[\mathrm{Fe} / \mathrm{H}] \sim-3$ ) is required for significant formation of low-mass protostellar aggregates.

It was found that the observed elemental abundances in stars with $-3 \leq[\mathrm{Fe} / \mathrm{H}]<-1$ could be quantitatively explained by the contributions from (1) the $P$ inventory at $[\mathrm{Fe} / \mathrm{H}] \approx-3$ for all the elements up to $\mathrm{Zr}$, (2) a high-frequency type of SNe II

\footnotetext{
${ }^{1}$ School of Physics and Astronomy, University of Minnesota, 116 Church Street, SE, Minneapolis, MN 55455; qian@physics.umn.edu.

${ }^{2}$ Department of Astronomy, California Institute of Technology, MS 10524, Pasadena, CA 91125.

${ }^{3}$ The Lunatic Asylum, Division of Geological and Planetary Sciences, California Institute of Technology, MS 170-25, Pasadena, CA 91125.
}

[SNe II $(H)]$ that are responsible for the heavy $r$-process elements and some light $r$-process elements below Ba but produce very little of the elements from $\mathrm{N}$ to $\mathrm{Zn}$ including $\mathrm{Fe}$, and (3) a lowfrequency type of SNe II [SNe II $(L)]$ that are responsible for the bulk of the light $r$-process elements and the part of the elements from N to Zn attributable to SNe II (Qian \& Wasserburg 2001b, 2002). It was further found by reducing the observational data on metal-poor Galactic halo stars that the yield patterns of SNe II $(L)$ and VMSs are almost the same for all the elements below $\mathrm{Sr}$ except for some small but significant shifts at $\mathrm{Na}, \mathrm{Al}$, $\mathrm{V}, \mathrm{Cr}, \mathrm{Mn}$, and Co. Thus, the abundance ratios of the so-called $\alpha$-elements relative to $\mathrm{Fe}$ such as $\mathrm{Mg} / \mathrm{Fe}, \mathrm{Si} / \mathrm{Fe}, \mathrm{Ca} / \mathrm{Fe}$, and $\mathrm{Ti} / \mathrm{Fe}$ remain essentially constant at $-4 \leqq[\mathrm{Fe} / \mathrm{H}]<-1$, although the dominant source of the $\alpha$-elements and Fe changes from VMSs in the regime of $[\mathrm{Fe} / \mathrm{H}]<-3$ to $\mathrm{SNe} \mathrm{II}(L)$ in the regime of $-3<[\mathrm{Fe} / \mathrm{H}]<-1$. These ratios are all greater than the corresponding solar values by a factor of $\sim 3$ because approximately two-thirds of the solar Fe inventory is from SNe Ia, which later added the $\mathrm{Fe}$ group elements but little of the $\alpha$ elements at $[\mathrm{Fe} / \mathrm{H}] \geq-1$. This simple explanation should serve to clarify the so-called overproduction of what are referred to as the $\alpha$-elements at low metallicities.

It was considered that the $P$ inventory at $[\mathrm{Fe} / \mathrm{H}] \approx-3$ is dominated by the contributions from VMSs with small contributions from SNe II. The rare occurrence of Galactic halo stars with $-4 \lesssim[\mathrm{Fe} / \mathrm{H}]<-3$, which must have masses of $\sim 1 M_{\odot}$ in order to survive to the present epoch, is considered to represent concomitant formation of some "normal" stars in the regime where VMSs dominated astration (Qian \& Wasserburg 2002). It was also suggested that VMSs were the source for reionization of the IGM (e.g., Qian \& Wasserburg 2001b). A recent study by Oh et al. (2001) showed that in producing a "metal" inventory corresponding to $[\mathrm{Fe} / \mathrm{H}] \sim-3$ (or $[\mathrm{Si} / \mathrm{H}] \sim-2.5$ ), VMSs also provide $\sim 1-10$ ionizing photons for $\mathrm{H}$ and $\mathrm{He}$. This was estimated from the VMS model yields calculated by Heger \& Woosley (2002) and the VMS emission spectra calculated by Bromm, Kudritzki, \& Loeb (2001b). It appears that by processing $\sim 10^{-5}$ to $10^{-4}$ of the baryonic matter, VMSs are capable of providing the $P$ inventory and also of reionizing the IGM prior to the formation of most galaxies. 
Further justification for the role of VMSs is found from the very low $\mathrm{Ba}$ abundances at $[\mathrm{Fe} / \mathrm{H}]<-3$. This requires that any Ba produced by $\mathrm{SNe}$ II at $[\mathrm{Fe} / \mathrm{H}]<-3$ had to be mixed in a dilution mass of $\sim 10^{6}-10^{7} M_{\odot}$, to be compared with the dilution mass of $\sim 3 \times 10^{4} M_{\odot}$ for $\mathrm{SNe}$ II at $[\mathrm{Fe} / \mathrm{H}]>-3$. It is considered that the severely disruptive VMS activities at $[\mathrm{Fe} / \mathrm{H}]<$ -3 would not often permit the preservation of baryonic aggregates with masses of $\lesssim 10^{6}-10^{7} M_{\odot}$ and that the $P$ inventory at $[\mathrm{Fe} / \mathrm{H}] \approx-3$ should represent the chemical composition of the general IGM (Qian \& Wasserburg 2002). Elemental abundances in the IGM are best measured from absorption lines produced by regions of enhanced density such as $\operatorname{Ly} \alpha$ systems that are illuminated by quasi-stellar objects (QSOs). For a given QSO, absorption lines occur for narrow ranges in redshift (below $z$ of the QSO), and the absorbing systems represent concentrations of dispersed baryonic matter between the QSO and the observer. Extensive studies have determined the abundances of $\mathrm{C}$ IV, $\mathrm{O}$ VI, and $\mathrm{Si}$ IV in $\mathrm{Ly} \alpha$ systems with a wide range in redshift from $z \sim 0.09$ up to $z \sim 5.3$. In the following, we will present the abundances of a large number of elements in the IGM based on the model of the $P$ inventory ( $(2)$ and compare these results with the available data on Ly $\alpha$ systems ( $\$ 3)$. The cosmological implications of our model will be discussed $(\$ 4)$.

\section{ELEMENTAL ABUNDANCES IN Ly $\alpha$ SYSTEMS AS DETERMINED FROM THE $P$ INVENTORY}

The abundances for the $P$ inventory are given in Table 1 in the standard spectroscopic notation as

$$
\log \epsilon_{P}(\mathrm{E})=\log \left(\frac{\mathrm{E}}{\mathrm{H}}\right)_{P}+12,
$$

where $(\mathrm{E} / \mathrm{H})_{P}$ is the number ratio of an element $\mathrm{E}$ relative to hydrogen for the $P$ inventory. The values of $\log \epsilon_{P}(\mathrm{E})$ in Table 1 are directly obtained from observational data on Galactic halo stars with $[\mathrm{Fe} / \mathrm{H}] \approx-3$ and correspond to those given in Qian \& Wasserburg (2002). The only element for which there may be a serious uncertainty is $\mathrm{O}$. The value of $\log \epsilon_{P}(\mathrm{O})$ assumed here is based on observations of HD 115444 and HD 122563 (Westin et al. 2000). Other extensive studies (e.g., Israelian, García López, \& Rebolo 1998; Boesgaard et al. 1999) indicate that $\log \epsilon_{P}(\mathrm{O})$ may be up to $\sim 0.3$ dex higher than the value given in Table 1 . The precise value of $\log \epsilon_{P}(\mathrm{O})$ is an important issue that remains to be resolved.

We consider that essentially all of the baryonic matter was ionized and dispersed in the IGM when VMS activities ceased and that the chemical composition of this baryonic matter was represented by the $P$ inventory. The mechanism for maintaining the ionized state of the IGM will not be discussed here. We assume that subsequent to the acquisition of the $P$ inventory, the bulk of the baryonic matter remains dispersed in the form of Ly $\alpha$ systems. We further assume that these systems have experienced no further evolution in elemental abundances and that they represent regions of enhanced density comoving with the expansion of the universe. Under these assumptions, the number ratio of an element $\mathrm{E}$ relative to $\mathrm{H}$ in the $\mathrm{Ly} \alpha$ systems is the same as that for the $P$ inventory and can be written as

$$
\left(\frac{\mathrm{E}}{\mathrm{H}}\right)_{P}=\frac{\Omega_{P}(\mathrm{E}) / m_{\mathrm{E}}}{\Omega(\mathrm{H}) / m_{\mathrm{H}}}=\frac{\Omega_{P}(\mathrm{E}) / A_{\mathrm{E}}}{\Omega(\mathrm{H})},
$$

where $\Omega_{P}(\mathrm{E})$ and $\Omega(\mathrm{H})$ are the fractions of the critical mass density $\rho_{\text {cri }}=3 H_{0}^{2} /(8 \pi G)$ contributed by E and $\mathrm{H}$, respectively, at the present epoch, $m_{\mathrm{E}}$ and $m_{\mathrm{H}}$ are the atomic masses of $\mathrm{E}$
TABLE 1

$\Omega_{P}($ E)-Values as Determined FROM THE $P$ INVENTORY

\begin{tabular}{crccc}
\hline \hline $\begin{array}{c}\mathrm{E} \\
(1)\end{array}$ & $\begin{array}{c}A_{\mathrm{E}} \\
(2)\end{array}$ & $\begin{array}{c}\log \epsilon_{P}(\mathrm{E}) \\
(3)\end{array}$ & $\begin{array}{c}\Omega_{P}(\mathrm{E}) \\
(4)\end{array}$ & $\begin{array}{c}{[\mathrm{E} / \mathrm{H}]} \\
(5)\end{array}$ \\
\hline $\mathrm{C} \ldots \ldots \ldots$ & 12 & $5.11^{\mathrm{a}}$ & $5.56 \times 10^{-8}$ & -3.45 \\
$\mathrm{~N} \ldots \ldots \ldots$ & 14 & 6.30 & $1.00 \times 10^{-6}$ & -1.75 \\
$\mathrm{O} \ldots \ldots \ldots$ & 16 & 6.60 & $2.29 \times 10^{-6}$ & -2.33 \\
$\mathrm{Na} \ldots \ldots$. & 23 & 3.34 & $1.81 \times 10^{-9}$ & -2.99 \\
$\mathrm{Mg} \ldots \ldots$. & 24 & 5.13 & $1.16 \times 10^{-7}$ & -2.45 \\
$\mathrm{Al} \ldots \ldots$. & 27 & 3.12 & $1.28 \times 10^{-9}$ & -3.35 \\
$\mathrm{Si} \ldots \ldots$. & 28 & 5.02 & $1.05 \times 10^{-7}$ & -2.53 \\
$\mathrm{Ca} \ldots \ldots$. & 40 & 3.75 & $8.09 \times 10^{-9}$ & -2.61 \\
$\mathrm{Sc} \ldots \ldots$. & 45 & 0.28 & $3.08 \times 10^{-12}$ & -2.82 \\
$\mathrm{Ti} \ldots \ldots$. & 48 & 2.43 & $4.65 \times 10^{-10}$ & -2.56 \\
$\mathrm{~V} \ldots \ldots \ldots$ & 51 & 1.15 & $2.59 \times 10^{-11}$ & -2.85 \\
$\mathrm{Cr} \ldots \ldots$. & 52 & 2.40 & $4.70 \times 10^{-10}$ & -3.27 \\
$\mathrm{Mn} \ldots \ldots$. & 55 & 1.90 & $1.57 \times 10^{-10}$ & -3.49 \\
$\mathrm{Fe} \ldots \ldots$. & 56 & 4.51 & $6.52 \times 10^{-8}$ & -3.00 \\
$\mathrm{Co} \ldots \ldots$. & 59 & 2.24 & $3.69 \times 10^{-10}$ & -2.68 \\
$\mathrm{Ni} \ldots \ldots$. & 58 & 3.25 & $3.71 \times 10^{-9}$ & -3.00 \\
$\mathrm{Cu} \ldots \ldots$. & 63 & 0.53 & $7.68 \times 10^{-12}$ & -3.68 \\
$\mathrm{Zn} \ldots \ldots \ldots$ & 64 & 1.86 & $1.67 \times 10^{-10}$ & -2.74 \\
$\mathrm{Sr} \ldots \ldots$. & 88 & 0.13 & $4.27 \times 10^{-12}$ & -2.77 \\
$\mathrm{Ba} \ldots \ldots$. & 138 & -1.80 & $7.87 \times 10^{-14}$ & -3.93 \\
\hline
\end{tabular}

Notes. - Col. (2): Approximate atomic mass number for the element. Col. (3): $P$ inventory. Col. (4): Fraction of the critical mass density contributed by the element at the present epoch. Col. (5): $[\mathrm{E} / \mathrm{H}]=\log \epsilon(\mathrm{E})-$ $\log \epsilon_{\odot}(\mathrm{E})$.

a The value of $\log \epsilon_{P}$ for $\mathrm{C}$ is obtained from data on HD 115444 and HD 122563 (Westin et al. 2000).

and $\mathrm{H}$, respectively, and $A_{\mathrm{E}}=m_{\mathrm{E}} / m_{\mathrm{H}}$ is the atomic mass number of E relative to hydrogen. The symbols $H_{0}$ and $G$ in the expression of $\rho_{\text {cri }}$ are the Hubble and gravitational constants, respectively. The baryonic contribution to $\rho_{\text {cri }}$ is found to be $\Omega_{b} \approx 0.02 h^{-2}$ based on measurements of deuterium abundances in QSO absorption systems (e.g., Burles, Nollett, \& Turner 2001) and the power spectrum of the cosmic microwave background (e.g., de Bernardis et al. 2002), where $h$ is $H_{0}$ in units of $100 \mathrm{~km} \mathrm{~s}^{-1} \mathrm{Mpc}^{-1}$. This gives $\Omega(\mathrm{H}) \approx X_{\mathrm{H}} \Omega_{b} \approx 0.0152 \mathrm{~h}^{-2}$, where $X_{\mathrm{H}} \approx 0.76$ is the mass fraction of $\mathrm{H}$. Combining equations (1) and (2), we obtain

$$
\Omega_{P}(\mathrm{E})=\Omega(\mathrm{H}) A_{\mathrm{E}} \times 10^{\log \epsilon_{P}(\mathrm{E})-12} \approx 1.52 h^{-2} A_{\mathrm{E}} \times 10^{\log \epsilon_{P}(\mathrm{E})-14} .
$$

Note that $\Omega_{P}(\mathrm{E})$ does not explicitly depend on cosmological models except for the numerical values of $H_{0}$ and $\Omega_{b}$. The values of $\Omega_{P}(\mathrm{E})$ calculated from the $P$ inventory are given in Table 1 for $h=0.65$. At present, data are available only for several elements such as $\mathrm{C}, \mathrm{O}$, and $\mathrm{Si}$ in $\mathrm{Ly} \alpha$ systems.

\section{COMPARISON WITH OBSERVATIONAL DATA}

Absorption lines produced by ions such as $\mathrm{C} \mathrm{IV,} \mathrm{O} \mathrm{VI,} \mathrm{and}$ $\mathrm{Si}$ IV in regions of enhanced density such as the Ly $\alpha$ systems have been measured in the spectra of QSOs that illuminate such regions. The column density of the ions of an element $\mathrm{E}$, $\mathrm{E}_{\mathrm{ion}}$, in a redshift interval $z_{-}<z<z_{+}$is

$$
N\left(\mathrm{E}_{\mathrm{ion}}\right)=c \int_{z_{-}}^{z_{+}} \frac{n\left(\mathrm{E}_{\mathrm{ion}}, z\right)}{(1+z) H(z)} d z,
$$

where $c$ is the speed of light, $n\left(\mathrm{E}_{\text {ion }}, z\right)$ is the number density of $\mathrm{E}_{\text {ion }}$ at redshift $z$, and $H(z)$ is the Hubble parameter at $z$. Under the assumptions that the bulk of the baryonic matter 
TABLE 2

IONIZATION FraCtIONS FOR Ly $\alpha$ SYSTEMS

\begin{tabular}{cccccc}
\hline \hline $\begin{array}{c}\mathrm{E} \\
(1)\end{array}$ & $\begin{array}{c}\Omega(\mathrm{E}) \\
(2)\end{array}$ & $\begin{array}{c}\mathrm{E}_{\text {ion }} \\
(3)\end{array}$ & $\begin{array}{c}\langle z\rangle \\
(4)\end{array}$ & $\begin{array}{c}\Omega\left(\mathrm{E}_{\text {ion }}\right) \\
(5)\end{array}$ & $\begin{array}{c}\Omega\left(\mathrm{E}_{\text {ion }}\right) / \Omega_{P}(\mathrm{E}) \\
(6)\end{array}$ \\
\hline $\mathrm{C} \ldots \ldots$ & $5.56 \times 10^{-8}$ & C IV & 1.87 & $2.05 \times 10^{-8}$ & 0.37 \\
& $5.56 \times 10^{-8}$ & C IV & 2.27 & $3.74 \times 10^{-8}$ & 0.67 \\
& $5.56 \times 10^{-8}$ & C IV & 2.78 & $2.62 \times 10^{-8}$ & 0.47 \\
& $5.56 \times 10^{-8}$ & C IV & 3.21 & $2.46 \times 10^{-8}$ & 0.44 \\
& $5.56 \times 10^{-8}$ & C IV & 3.75 & $3.68 \times 10^{-8}$ & 0.66 \\
& $5.56 \times 10^{-8}$ & C IV & 4.24 & $3.93 \times 10^{-8}$ & 0.71 \\
& $5.56 \times 10^{-8}$ & C IV & 4.66 & $1.35 \times 10^{-8}$ & 0.24 \\
& $5.56 \times 10^{-8}$ & C IV & 5.29 & $4.93 \times 10^{-9}$ & 0.09 \\
$\mathrm{O} \ldots \ldots$ & $2.29 \times 10^{-6}$ & O VI & 0.09 & $4.6 \times 10^{-7}$ & 0.20 \\
& $2.29 \times 10^{-6}$ & O VI & $\approx 0.24$ & $1 \times 10^{-6}$ & 0.44 \\
& $2.29 \times 10^{-6}$ & O VI & 0.9 & $\geq 1.3 \times 10^{-7}$ & $\geq 0.06$ \\
& $2.29 \times 10^{-6}$ & O VI & $\approx 2.5$ & $2.96 \times 10^{-7}$ & 0.13 \\
$\mathrm{Si} \ldots \ldots$ & $1.05 \times 10^{-7}$ & Si IV & 2.24 & $9.04 \times 10^{-9}$ & 0.09 \\
& $1.05 \times 10^{-7}$ & Si IV & 2.81 & $1.59 \times 10^{-8}$ & 0.15 \\
& $1.05 \times 10^{-7}$ & Si IV & 3.19 & $6.19 \times 10^{-9}$ & 0.06 \\
& $1.05 \times 10^{-7}$ & Si IV & 3.70 & $1.59 \times 10^{-8}$ & 0.15 \\
& $1.05 \times 10^{-7}$ & Si IV & 4.28 & $1.63 \times 10^{-8}$ & 0.16 \\
& $1.05 \times 10^{-7}$ & Si IV & 4.64 & $4.66 \times 10^{-9}$ & 0.04 \\
& $1.05 \times 10^{-7}$ & Si IV & 5.29 & $9.31 \times 10^{-10}$ & 0.01 \\
\hline
\end{tabular}

Notes. - Data on C IV and Si IV are from Songaila 2001, and data on O VI are from Savage et al. $2002(\langle z\rangle=0.09)$, Tripp et al. $2000(\langle z\rangle \approx 0.24)$, Burles \& Tytler $1996(\langle z\rangle=0.9)$, and Simcoe, Sargent, \& Rauch 2002 $(\langle z\rangle \approx 2.5)$. The $\Omega\left(\mathrm{E}_{\text {ion }}\right)$-values in col. (5) are recalculated from the published data so that these values correspond to a flat cosmological model with $\Omega_{m}=0.3, \Omega_{\Lambda}=0.7$, and $h=0.65$. Col. (2): Fraction of the critical mass density contributed by the element. Col. (4): Average redshift of the Ly $\alpha$ systems from which col. (5) is obtained. Col (5): Fraction of the critical mass density contributed by the ions of the element. Col. (6): Ionization fraction $f\left(\mathrm{E}_{\text {ion }}\right)=\Omega\left(\mathrm{E}_{\text {ion }}\right) / \Omega_{P}(\mathrm{E})$ estimated from the $P$ inventory.

resides in Ly $\alpha$ systems and that these systems experience no evolution other than comoving with the expansion of the universe, we obtain

$$
N\left(\mathrm{E}_{\mathrm{ion}}\right)=\frac{c n_{0}\left(\mathrm{E}_{\mathrm{ion}}\right)}{H_{0}} \int_{z_{-}}^{z_{+}} \frac{(1+z)^{2}}{H(z) / H_{0}} d z,
$$

where $n_{0}\left(\mathrm{E}_{\mathrm{ion}}\right)=n\left(\mathrm{E}_{\mathrm{ion}}, z\right) /(1+z)^{3}$ is the number density of $\mathrm{E}_{\mathrm{ion}}$ at the present epoch. By defining (Bahcall \& Peebles 1969)

$$
X(z) \equiv \int_{0}^{z} \frac{\left(1+z^{\prime}\right)^{2}}{H\left(z^{\prime}\right) / H_{0}} d z^{\prime}
$$

and $\Delta X \equiv X\left(z_{+}\right)-X\left(z_{-}\right)$, equation (5) can be rewritten as

$$
N\left(\mathrm{E}_{\mathrm{ion}}\right)=\frac{c n_{0}\left(\mathrm{E}_{\mathrm{ion}}\right)}{H_{0}} \Delta X
$$

If absorption lines due to $\mathrm{E}_{\mathrm{ion}}$ have been measured for a statistical sample of Ly $\alpha$ systems at different redshift along the lines of sight for a number of QSOs, the terms $N\left(\mathrm{E}_{\mathrm{ion}}\right)$ and $\Delta X$ in equation (7) are replaced by the sums $\sum N\left(\mathrm{E}_{\text {ion }}\right)$ and $\sum \Delta X$ over the sampled region. It follows that the fraction of $\rho_{\text {cri }}$ contributed by $\mathrm{E}_{\text {ion }}$ at the present epoch, $\Omega\left(\mathrm{E}_{\mathrm{ion}}\right)=$ $n_{0}\left(\mathrm{E}_{\text {ion }}\right) m_{\mathrm{E}} / \rho_{\text {cri }}$, is

$$
\begin{aligned}
\Omega\left(\mathrm{E}_{\mathrm{ion}}\right) & =\frac{H_{0}}{c \rho_{\text {cri }}} \frac{\sum N\left(\mathrm{E}_{\text {ion }}\right)}{\sum \Delta X} m_{\mathrm{E}} \\
& =9.55 \times 10^{-24} h^{-1} A_{\mathrm{E}} \frac{\sum N\left(\mathrm{E}_{\mathrm{ion}}\right)}{\sum \Delta X},
\end{aligned}
$$

where the last equality is obtained for $\sum N\left(\mathrm{E}_{\text {ion }}\right)$ in units of $\mathrm{cm}^{-2}$. If the model of the $P$ inventory is correct, the value of $\Omega\left(\mathrm{E}_{\mathrm{ion}}\right)$ obtained from equation (8) is related to that of $\Omega_{P}(\mathrm{E})$ from equation (3) through the ionization fraction $f\left(\mathrm{E}_{\mathrm{ion}}\right)=$ $\Omega\left(\mathrm{E}_{\text {ion }}\right) / \Omega_{P}(\mathrm{E})$.

The calculation of $\Omega\left(\mathrm{E}_{\mathrm{ion}}\right)$ through $\Delta X$ requires a specific cosmological model. This is distinct from the more direct calculation of $\Omega_{P}(\mathrm{E})$. The choice of a particular model makes little difference for $z \ll 1$, as all models give $\Delta X \approx z_{+}-z_{-}$in this limit. For simplicity in considering the data on $\operatorname{Ly} \alpha$ systems with a wide range in $z$, we adopt a flat cosmological model with $\Omega_{m}+\Omega_{\Lambda}=1$, where $\Omega_{m}$ and $\Omega_{\Lambda}$ are the fractions of $\rho_{\text {cri }}$ contributed by matter (baryonic and nonbaryonic) and the cosmological constant, respectively, at the present epoch. With this model, $\left[H(z) / H_{0}\right]^{2}=\Omega_{m}(1+z)^{3}+\Omega_{\Lambda}$ and the expression of $X(z)$ from equation (6) is

$$
X(z)=\frac{2}{3 \Omega_{m}}\left[\sqrt{\Omega_{m}(1+z)^{3}+1-\Omega_{m}}-1\right],
$$

which gives $\Delta X \approx z_{+}-z_{-}$for $z \ll 1$ and $\Delta X \approx \frac{2}{3} \Omega_{m}^{-1 / 2}[(1+$ $\left.\left.z_{+}\right)^{3 / 2}-\left(1+z_{-}\right)^{3 / 2}\right]$ for $1+z \gg\left[\left(1-\Omega_{m}\right) / \Omega_{m}\right]^{1 / 3}$.

A comparison of the observed values of $\Omega\left(\mathrm{E}_{\mathrm{ion}}\right)$ with the values corresponding to the $P$ inventory requires that the same cosmological model and parameters be used. The data on Ly $\alpha$ systems with a wide range in $z$ as reported in the literature were based on somewhat different cosmological models and parameters. We have recalculated $\Omega\left(\mathrm{E}_{\text {ion }}\right)$ from the published data so that the recalculated results correspond to the flat cosmological model adopted here with $\Omega_{m}=0.3, \Omega_{\Lambda}=0.7$, and $h=0.65$. The data on C IV and Si IV are taken from Songaila (2001) and those on O vi from Burles \& Tytler (1996), Tripp, Savage, \& Jenkins (2000), Savage et al. (2002), and Simcoe, Sargent, \& Rauch (2002; no recalculation necessary). The recalculated $\Omega\left(\mathrm{E}_{\text {ion }}\right)$-values are given in Table 2 . Ideally, the fraction $f\left(\mathrm{E}_{\text {ion }}\right)$ of the element $\mathrm{E}$ that is ionized to the particular state observed can be calculated for the Ly $\alpha$ systems from which $\Omega\left(\mathrm{E}_{\mathrm{ion}}\right)$ is obtained. A comparison can then be made of $\Omega(\mathrm{E})=\Omega\left(\mathrm{E}_{\text {ion }}\right) / f\left(\mathrm{E}_{\text {ion }}\right)$, with $\Omega_{P}(\mathrm{E})$ calculated from the $P$ inventory. As values of $f\left(\mathrm{E}_{\text {ion }}\right)$ are not always well known, we have tabulated the ratio $\Omega\left(\mathrm{E}_{\text {ion }}\right) / \Omega_{P}(\mathrm{E})$ for C IV, O VI, and $\mathrm{Si}$ IV in Table 2. This ratio should correspond to $f\left(\mathrm{E}_{\mathrm{ion}}\right)$ if the model of the $P$ inventory is valid. The values of $\Omega\left(\mathrm{E}_{\mathrm{ion}}\right) / \Omega_{P}(\mathrm{E})$ in Table 2 are in reasonable agreement with $f(\mathrm{C}$ IV $) \sim 0.5, f(\mathrm{O}$ VI $) \sim 0.2$, and $f(\mathrm{Si}$ IV $) \sim 0.1$ as estimated from various ionization models for Ly $\alpha$ systems.

For all the data sets used in the above comparison, there may be significant uncertainties in estimates of both $\sum N\left(\mathrm{E}_{\text {ion }}\right)$ and $\sum \Delta X$ due to sampling problems. The mechanisms of ionization are also not well understood. Considering the very different and complex data sets used and the extreme simplicity of our model for the $P$ inventory, we conclude that there is remarkable agreement between the observations and the predictions for the abundances in the IGM. We note that the apparently high values of $(\mathrm{O} / \mathrm{H}) /(\mathrm{O} / \mathrm{H})_{\odot}$ and $(\mathrm{C} / \mathrm{H}) /(\mathrm{C} / \mathrm{H})_{\odot}$ reported by Burles \& Tytler (1996) are off owing to the omission of a factor $1 / A_{\mathrm{E}}$ in their equation for $\zeta(z)$ (D. Tytler 2002, private communication). We also note that the values of $[\mathrm{O} / \mathrm{H}]$ used by Tripp et al. (2000) and Savage et al. (2002) in estimating the lower bounds to $\Omega_{b}$ may not be appropriate in view of the results presented here. We consider that a more detailed comparison of the model for the $P$ inventory and the observations must await the availability of more complete data sets with appropriate estimates of errors including those in the term $\sum \Delta X$. It would be most useful to theoretical studies of $\operatorname{Ly} \alpha$ systems if observational reports in- 
cluded explicit statements on the value of $\sum N\left(\mathrm{E}_{\mathrm{ion}}\right)$ and on the detailed redshift intervals for the calculation of $\sum \Delta X$ with corrections for the obscured part of the redshift path. We note that compared with the flat cosmological model with $\Omega_{m}=0.3$ and $\Omega_{\Lambda}=0.7$ as adopted here, a flat model with $\Omega_{m}=1$ (i.e., with the deceleration parameter $\left.q_{0}=0.5\right)$ gives smaller $\Delta X$-values, and hence larger $\Omega\left(\mathrm{E}_{\mathrm{ion}}\right)$-values, by a factor of 1.83 at high $z$. It is possible that the model for the $P$ inventory may be of use in evaluating various cosmological models if the ionization mechanism for Ly $\alpha$ systems is better understood.

\section{DISCUSSION}

The results in Table 2 strongly support our assumptions that subsequent to the acquisition of the $P$ inventory, the bulk of baryonic matter remains dispersed in the form of $\operatorname{Ly} \alpha$ systems and that these systems have experienced little or no chemical evolution but are simply comoving with the expansion of the universe. It is striking to note that these assumptions hold from $z \sim 2.5$ down to $\sim 0.09$ based on the $\mathrm{O}$ VI data and from $z \sim$ 4.6 down to $\sim 2$ based on the $\mathrm{C}$ IV and Si IV data. We conclude that the bulk of ionized baryonic matter in the IGM has not been greatly diminished from the epoch of reionization until the present epoch.

We have argued that VMSs are responsible for the $P$ inventory and reionization of the IGM. An important issue is the timescale over which this is achieved. From the range in $z$ where the model appears to describe the abundances observed in Ly $\alpha$ systems, we infer that the IGM had already acquired the $P$ inventory before $z \sim 4.6$. This is consistent with the following considerations of the cosmological epoch during which VMSs could disperse processed baryonic matter to the general IGM. The explosion energies of VMSs are $\sim 10^{52}-10^{53}$ ergs, as estimated from the available models of Heger \& Woosley (2002). Based on the cold dark matter model of structure formation, dark matter halos corresponding to $1 \sigma$ fluctuations and collapsing at $z \sim 4$ or those corresponding to $2 \sigma$ fluctuations and collapsing at $z \sim 10$ provide binding energies of $\sim 10^{52}-10^{53}$ ergs for the baryonic matter in their potential wells (see Fig. 9 in Barkana \& Loeb 2001). Thus, VMS explosions could readily unbind baryonic matter that was not in stars from most dark matter halos formed at $z>4$. We conclude that VMSs dominate both the elemental production and the dispersal of processed baryonic matter at $z>4$. Compared with the nearly constant values of $\Omega(\mathrm{C}$ IV) and $\Omega(\mathrm{Si}$ IV $)$ at $z \sim 2-4.6$, the apparent decrease in these two quantities from $z \sim 4.6$ to $\sim 5.3$ (see Table 2 here or Table 1 in Songaila 2001) may be a hint for cessation of VMS activities at $z \sim 4.6$, although this decrease may also be due to the incompleteness of the sample of $\operatorname{Ly} \alpha$ systems in the highest redshift interval. Songaila \& Cowie (2002) have reported a damped $\mathrm{Ly} \alpha$ system with $[\mathrm{Fe} / \mathrm{H}] \approx-2.7$ and $z=5.3$. This indicates that VMS activities may have ceased as early as $z \sim$ 5.3. In any case, the bulk of baryonic matter should have been enriched with the $P$ inventory, ionized, and dispersed in the IGM when the epoch of VMS activities ended. The subsequent reaggregation of baryonic matter into galaxies appears to be very inefficient, as demonstrated by the prevalence of the $P$ inventory in Ly $\alpha$ systems with $z \sim 0.09-4.6$. When galaxies do form, it appears that the times of formation vary greatly, as argued by Wasserburg \& Qian (2000b) based on the large scatter in $[\mathrm{Fe} / \mathrm{H}]$ for damped Ly $\alpha$ systems at a given redshift in the range of $z \approx 1.5-4.5$. The precise epoch of VMS activities and its influence on structure formation at later times are important issues that remain to be addressed.

We would like to dedicate this Letter to the memory of Fritz Zwicky. We thank Antoinette Songaila, Todd Tripp, and David Tytler for generously answering many questions and for guiding us through their data. This work was supported in part by the Department of Energy under grants DE-FG02-87ER40328 and DE-FG02-00ER41149, by the NSF under grant AST 9900733, and by NASA under grant NAG5-10293, Caltech division contribution $8780(1091)$.

\section{REFERENCES}

Bahcall, J. N., \& Peebles, P. J. E. 1969, ApJ, 156, L7

Barkana, R., \& Loeb, A. 2001, Phys. Rep., 349, 125

Boesgaard, A. M., King, J. R., Deliyannis, C. P., \& Vogt, S. S. 1999, AJ, 117, 492

Bromm, V., Ferrara, A., Coppi, P. S., \& Larson, R. B. 2001a, MNRAS, 328, 969

Bromm, V., Kudritzki, R. P., \& Loeb, A. 2001b, ApJ, 552, 464

Burles, S., Nollett, K. M., \& Turner, M. S. 2001, ApJ, 552, L1

Burles, S., \& Tytler, D. 1996, ApJ, 460, 584

Burris, D. L., Pilachowski, C. A., Armandroff, T. E., \& Sneden, C. 2000, ApJ, 544,302

de Bernardis, P., et al. 2002, ApJ, 564, 559

Heger, A., \& Woosley, S. E. 2002, ApJ, 567, 532

Israelian, G., García López, R. J., \& Rebolo, R. 1998, ApJ, 507, 805

McWilliam, A., Preston, G. W., Sneden, C., \& Searle, L. 1995, AJ, 109, 2757

Oh, S. P., Nollett, K. M., Madau, P., \& Wasserburg, G. J. 2001, ApJ, 562,
Prochaska, J. X., Gawiser, E., \& Wolfe, A. M. 2001, ApJ, 552, 99

Prochaska, J. X., \& Wolfe, A. M. 2000, ApJ, 533, L5

Qian, Y.-Z., \& Wasserburg, G. J. 2001a, ApJ, 549, 337 2001b, ApJ, 559, 925

2002, ApJ, 567, 515

Savage, B. D., Sembach, K. R., Tripp, T. M., \& Richter, P. 2002, ApJ, 564, 631

Simcoe, R. A., Sargent, W. L. W., \& Rauch, M. 2002, ApJ, submitted

Songaila, A. 2001, ApJ, 561, L153

Songaila, A., \& Cowie, L. L. 2002, AJ, in press

Tripp, T. M., Savage, B. D., \& Jenkins, E. B. 2000, ApJ, 534, L1

Wasserburg, G. J., \& Qian, Y.-Z. 2000a, ApJ, 529, L21 2000b, ApJ, 538, L99

Westin, J., Sneden, C., Gustafsson, B., \& Cowan, J. J. 2000, ApJ, 530, 783 